REVISED Manuscript

\title{
Exploring the "Resistance Change per Energy Unit" as Universal Performance Parameter for Resistive Switching Devices
}

\author{
J. Gomez ${ }^{1}$, I. Vourkas ${ }^{2 *}$, A. Abusleme ${ }^{1}$, R. Rodríguez ${ }^{3}$, J. Martin-Martinez ${ }^{3}$, M. Nafria ${ }^{3}$, and A. \\ Rubio $^{4}$ \\ ${ }^{1}$ Dept. of Electrical Engineering, Pontificia Universidad Católica de Chile, Santiago, CHILE \\ ${ }^{2}$ Dept. of Electronic Engineering, Universidad Técnica Federico Santa Maria, Valparaiso, CHILE \\ ${ }^{3}$ Dept. of Electronic Engineering, Universitat Autònoma de Barcelona, Barcelona, SPAIN \\ ${ }^{4}$ Dept. of Electronic Engineering, Universitat Politècnica de Catalunya, Barcelona, SPAIN \\ *Corresponding author email: ioannis.vourkas@usm.cl
}

\begin{abstract}
Resistive switching (RS) device (memristor) technology is continuously maturing towards industrial establishment. There are RS devices that demonstrate an "incremental" (analog) switching behavior, whereas others change their state in a binary form. The final achieved resistance is generally a function of the applied pulse characteristics, i.e. amplitude and duration. However, variability - both from device to device but also from cycle to cycle - and the stochastic nature of internal RS phenomena, still hold back any universal tuning approach based solely on these two magnitudes, making also difficult the qualitative comparison between devices with different material compounds owing to the SET/RESET voltages being dependent on the biasing conditions. In this work we demonstrate experimentally using commercial RS devices from Knowm Inc. that the switching energy is not very sensitive to the biasing conditions. We explored experimentally the SET-RESET behavior of bipolar RS devices from the energy point of view. We figured out the quantitative effect of the injected energy to the resistive state of the devices, and proposed an analytical model to explain our observations in the energy consumption during the switching process. Our results lay the foundations for the definition of "resistance change per energy unit" as a performance parameter for this emerging device technology.
\end{abstract}

Keywords: memristor; resistive switching; ReRAM; device characterization; Knowm; energy consumption.

\section{Introduction}

The introduction of resistive switching (RS) devices (also called memristors) to the set of standard electronic components is expected to enrich significantly the landscape of modern circuit design and development [1-5]. The memristor has been known as the fourth fundamental circuit element ever since Leon Chua postulated its existence in 1971 [6]. However, a lot of attention was drawn on this new device technology only after 2008 with the demonstration of the $\mathrm{TiO}_{2}$-based memristor by a team of scientists at Hewlett Packard (HP) [7], [8], who managed to connect the nature of such RS devices with Chua's theory. During the last ten years, memristors created new R\&D tracks and trends in modern circuits \& systems, essentially being electrically tunable, multi-level and non-volatile resistive elements with CMOS Back-End-of-Line (BEOL) integration-compatibility. Such properties render memristors promising candidates for use in several applications including memory [9], logic [10] and unconventional computing [11], [12]. 


\section{REVISED Manuscript}

The HP Labs' discovery also included the development of a simple device model, which has been ever since the basis for several other models, either behavioral or physics-based, which go deeper into the device dynamics [13-15] aiming to explain the mechanisms that control the change of resistance. Such activity reveals the important steps made for the continuous advancement of this technology towards industrial production, with some devices being already commercially available [16].

RS devices can be either unipolar or bipolar [17] and are usually fabricated in a metal-insulator-metal (MIM) structure. The RS property is observed in the insulating material [18], [19]. However, different materials can behave in a different way when exposed to the electrical field induced by the applied voltage. For instance, filamentary devices generally show sharp conductance transitions whereas interfacial (or homogeneous) switching devices tend to respond more slowly to the applied input signals, thus modifying their state in a more incremental manner [20]. Operation of the latter depends on the proper selection of the applied programming (SET or RESET) pulse, as they can still act as digital switches for long/high-enough applied pulses.

In this context, as far as device characterization is concerned, the change of resistance has been identified as a function of the properties of the applied pulse, such as amplitude and length. However, parameter variability, which is still observed in all such devices (e.g. in the voltage thresholds for SET and RESET processes), holds back any universal modeling approach to the switching behavior based solely on the programming pulse amplitude and length. In other words, due to the stochastic nature of internal RS phenomena [21], it is difficult to drive the device accurately and repeatedly to a specific resistance just by applying a single and well-calculated (SET/RESET) pulse. Therefore, the lack of any such unifying modeling approach renders difficult even the qualitative comparison/selection between devices built out of different material compounds that exhibit RS behavior. Nevertheless, it was recently demonstrated in [22] that there is a relevant and comprehensive magnitude which is not much affected by the biasing conditions and thus demonstrates low variability; the energy injected into the device during a resistive state transition. So, using the energy as primary parameter, a first attempt towards the definition of some key performance parameters was made for unipolar RS devices in [23], with an emphasis on the "probability change per energy unit" ( $d P / d E)$ for binary RS mode. Such parameter describes how probable a binary RS event is depending on the total energy injected into the device under test.

In this direction, this manuscript extends the ideas and the preliminary findings presented in [24] about bipolar RS devices. Our experimental results on commercial bipolar RS devices by Knowm Inc. confirm that the energy, and particularly the "resistance change per energy unit" $(d R / d E)$, is a comprehensive magnitude, suitable not only for the behavioral analysis of the analog operation of memristors, but also to be used as a universal performance parameter capable of characterizing quantitatively different RS device families. We corroborate this property by observing the trends in the switching behavior using experimental data taken on totally different RS devices (in terms of material structure and switching mechanism). Moreover, we show that the cumulative effect of a specific amount of energy on the state of the device is on average the same, regardless of the way this energy was injected; i.e. regardless of the properties of the applied pulses. Finally, we propose an analytical model attributing the energy consumed by the RS device during the switching process to 


\section{REVISED Manuscript}

Joule heating and to a critical energy required to induce the resistance change, with very good quantitative matching with the experimental data.

\section{Experimental Results}

\section{- 2.1 Experimental Methods \& Setup}

Unless mentioned differently in the manuscript, all measurements were carried out in a fully automated manner using the digital oscilloscope and the function generator of the Digilent Analog Discovery 2 (AD2) instrumentation tool [25] with real-time monitoring of data and using custom PCBs that connect directly to AD2. The memristor bottom electrode was connected to the inverting input of the operational amplifier in a transinpedance amplifier topology which enables: (i) controlling the voltage drop on the memristor terminals, (ii) sensing the current through it via a current to voltage conversion, and (iii) adjusting the compliance current to protect the memristor from damage (for further details on the setup see [24], [26]). All RS devices used were BS-AF-W discrete self-directed-channel (SDC) bipolar memristors [27] with tungsten (W) as dopant on a chalcogenide material, developed and commercialized in 16-pin ceramic DIP packages by Knowm Inc. in collaboration with Boise State University [16]. Such devices operate primarily through the electric field-induced generation of metal (Ag) ions that move through a multilayer chalcogenide material stack to change the device resistance. Once the ions reach the lower potential electrode, they are reduced to their metallic form and eventually create a conductive channel that spans the active material layer. The amount of Ag within the channels determines the resistance of the device. Knowm Inc. SDC memristors are distinct from Conductive Bridge RAM (CBRAM) technology and represent their own class of metal-ion memristors.

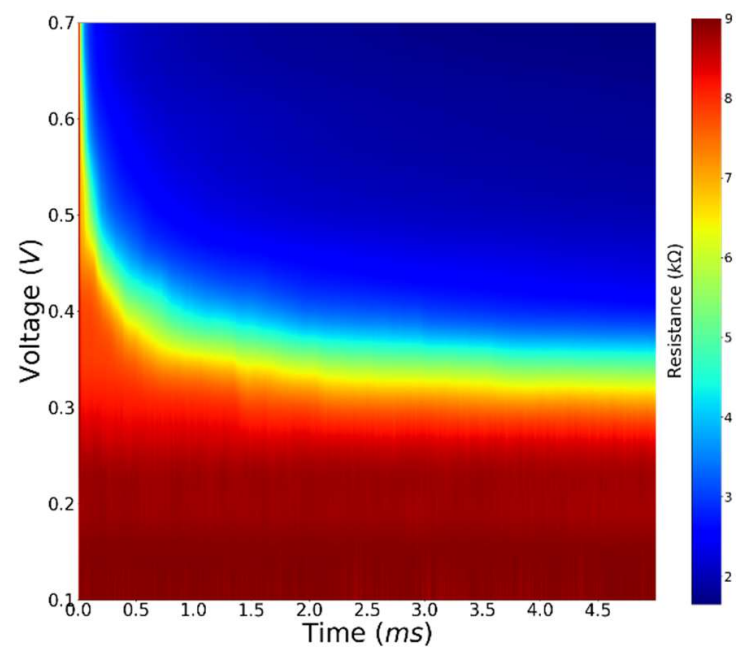

(a)

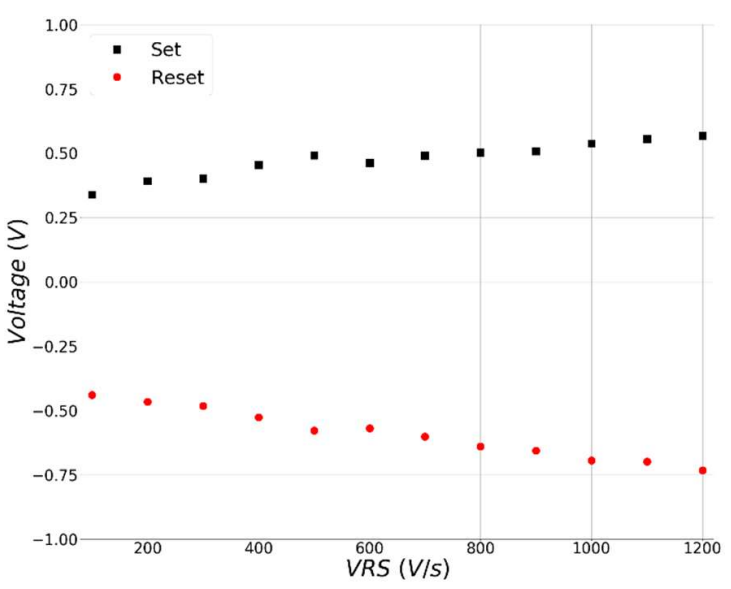

(b)

Fig. 1 (a) Dependence of resistance evolution on the amplitude and the width of the applied SET voltage pulse. The device was first initialized around $8.5 \mathrm{k} \Omega .5$-ms wide pulses of amplitude between $0.1 \mathrm{~V}$ and $0.7 \mathrm{~V}$ (incrementing by $40 \mathrm{mV}$ ) were applied. For each amplitude, the experiment was repeated $10 \times$ and the mean values are shown (interpolated). (b) Average required voltage to reach to a specific resistance value during SET/RESET, depending on the applied voltage ramp speed (VRS). Every point in the plot corresponds to the average of 10 experiments realized for each one of the 12 VRS values. Figures adapted from [24]. 


\section{REVISED Manuscript}

\section{- 2.2 Resistance Tuning as Function of Pulse Amplitude/Duration}

As mentioned previously, the change of resistance has been identified as a function of the properties of the applied pulse, i.e. its amplitude and duration. That said, in our study we first focused on pulsed SET/RESET inputs in order to analyze the impact of the pulse properties on the device behavior. Fig. 1(a) reveals such dependencies of resistance change on the pulse amplitude and on time during the SET process. More specifically, a precise RESET (implementing the tuning algorithm suggested in [28]) took place before each SET experiment. Then, a 5-ms wide SET pulse of a specific amplitude was applied and the time-evolution of the resistance was monitored. The same experiment was repeated several times and for different amplitude values. Fig. 1(a) shows the memristance timeevolution for different pulse amplitudes. There is a clear separation (approximately around $0.35 \mathrm{~V}$ ) between amplitudes that hardly affect the device state and those that do affect it. So, the target bipolar RS devices used in this work demonstrate incremental threshold-based switching behavior. Moreover, such data permit selecting the appropriate amplitude and width of the applied pulses depending on the target task. For instance, pulses with amplitudes higher than $0.6 \mathrm{~V}$ would serve mostly for binary switching. On the other hand, amplitudes between $0.3 \mathrm{~V}$ and $0.5 \mathrm{~V}$ permit incremental multi-level tuning, whereas lower amplitudes can be used to sense the device state without disturbing it. Qualitatively similar results can be obtained for the RESET process (not shown here). Besides the threshold-based behavior, Fig. 1(a) shows that the final achieved resistance indeed depends not only on the selected pulse amplitude but also on its duration. This behavior is well-known and frequently mentioned in relevant works in the literature. Our results confirm that pulses of lower amplitude need more time to drive the device to a specific resistance, than pulses of higher amplitude.

This is further highlighted in Fig. 1(b), which shows results for a set of 240 total (120 positive and 120 negative) voltage ramps applied for SET and RESET, with different voltage-ramp-speed (VRS) ranging from $100 \mathrm{~V} / \mathrm{s}$ to $1200 \mathrm{~V} / \mathrm{s}$, increasing each time by $100 \mathrm{~V} / \mathrm{s}$. Prior to the realization of every experiment, the device was gradually SET or RESET always in the same way to eliminate any dependence of the result on the initial conditions. The voltage and current were monitored throughout each experiment. Fig. 1(b) shows the average voltage when the device completed the SET or RESET process for every VRS value. In this context, we assumed that SET/RESET was successful when the resistance was below $2 \mathrm{k} \Omega$ /above $3 \mathrm{k} \Omega$, respectively, leaving in-between a 1-k $\Omega$ wide guard band (as undefined state). Such resistive thresholds were selected arbitrarily in the resistance window where the devices were operated. It can be observed that, the higher the VRS, the higher the absolute voltage required so that the device can reach the target resistance during SET or RESET. Similar results were demonstrated in [22] for the switching thresholds of unipolar $\mathrm{Ni} / \mathrm{HfO}_{2}$ based devices while modifying VRS. Such findings indicate that selecting the programming pulse amplitude for multi-level resistance tuning while solely considering its length, might not be a correct strategy to follow, being also susceptible to variability in switching behavior. Moreover, the dependence of the required voltage on VRS shown here and also in [22], suggests the existence of a critical energy required to induce the SET/RESET process.

\section{- 2.3 Resistance Tuning from the Energy Point of View}

The energy required to cause the RS event is indeed a magnitude that unifies such exploratory analysis, as it combines the effect of voltage and time simultaneously. Moreover, it could be explained physically as every conductive channel requires a minimum amount of energy to be created/dissolved. 


\section{REVISED Manuscript}

Next we show that the energy generally demonstrates low variability and it is independent of the biasing conditions. More specifically, we computed the accumulated energy required to reach a specific resistance value during SET/RESET depending on the applied VRS. We computed the energy by integrating the instantaneous power over time until the SET/RESET was complete, focusing exclusively on the device and assuming the considered voltage is applied directly to the device terminals, without taking into account the consumption in the driving circuitry. By observing Fig. 2 we notice that the average energy required during a SET process is nearly constant, independent of the applied VRS. The same applies for the RESET, but only for high VRS values; i.e., for very sharp voltage ramp inputs. This is because, for the slowly increasing ramps, especially during the RESET process when the device is initially in a low resistive state, there is a significant amount of energy consumed by the device even before we start noting a change in its state [see also next Fig. 5(a)]. This is why the measured energy for RESET increases as VRS decreases. It should be also considered that for a specific VRS value, the average absolute voltage required to complete the RESET process is always higher than for the SET process, according to Fig. 1(b), which means that during RESET it takes longer to initiate switching and thus to overshoot the $3-\mathrm{k} \Omega$ resistive threshold mentioned previously. In the following sections we will get back to this observation to explain further these results through the development of an analytical model for the energy delivered to the device.

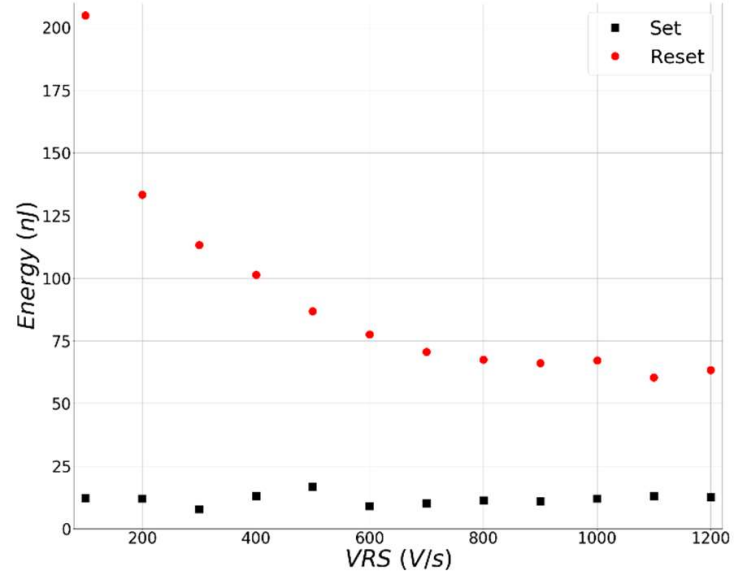

Fig. 2 Average SET/RESET energy required to trigger the RS event, depending on the applied voltage ramp speed (VRS). Every point in the plot corresponds to the average of 10 experiments realized for each one of the 12 different VRS values. Adapted from [24].

These results for the energy consumed by the device are qualitatively very much in line with results published previously in [22], where a totally different unipolar device family was considered. Hence, the energy indeed appears to be a good candidate for a universal parameter to be considered for such performance analysis. Studying the RS behavior from the energy point of view can also reveal the energy-efficiency of such device technology, required for modern memory and computing applications. Therefore, we computed the average energy cost of RS events, which allowed us to figure out the quantitative effect of the injected energy on the resistive state of a device. In this context, in Fig. 3(a) we show the histogram of the total resistance change per energy unit calculated for a total of 100 SET events while applying voltage ramps with a VRS of 1000V/s shown in Fig. 3(c). For threshold-based switching behavior, the maximum voltage of the applied ramp should exceed the respective voltage thresholds, according to results shown in Fig. 1. Besides cycle-to-cycle variability, 


\section{REVISED Manuscript}

it can be observed that the "resistance change per energy unit" $(d R / d E)$ values are very concentrated around a mean value (shown with a vertical dashed line). The same was observed in other similar experiments regardless of the applied VRS. Based on such analysis, the $d R / d E$ performance parameter could be extracted for any RS device family, to be used for comparison purposes and also in device modeling tasks.

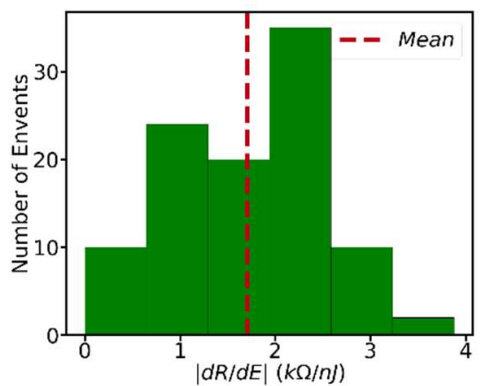

(a)

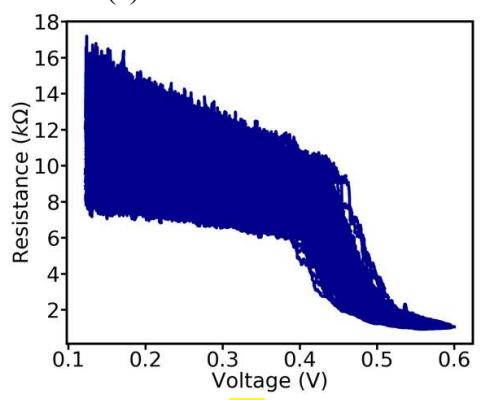

(d)

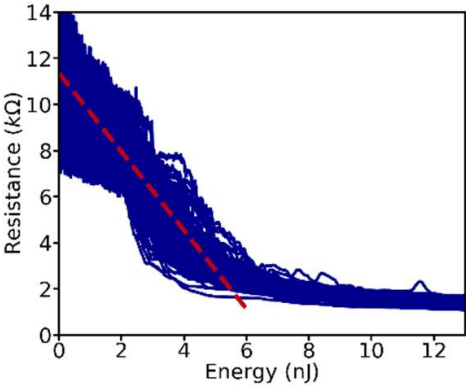

(b)

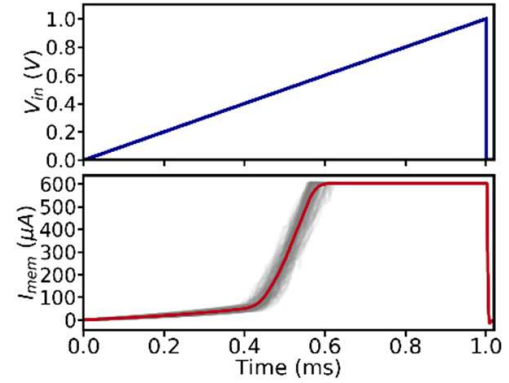

(c)

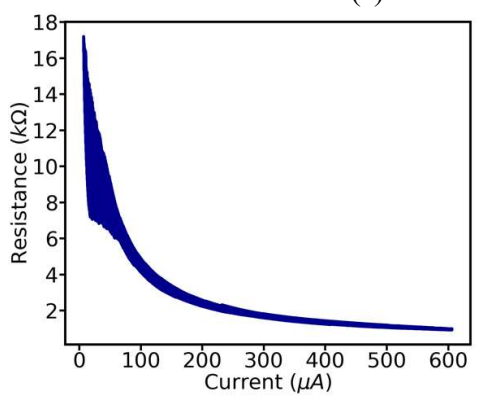

(e)

Fig. 3 Performance parameter analysis for analog operation of Knowm Inc. memristors under ramp voltage. (a) Histogram of 100 SET events depending on the resistance change per energy unit $(d R / d E)$ value. The vertical dashed line shows the mean value. (b) The corresponding resistance evolution w.r.t. the injected energy during the 100 SET events, applying a $1000 \mathrm{~V} / \mathrm{s}$ VRS. The dashed line shows a linear approximation fit, based on the extracted $d R / d E$ parameter from (a). (c) The applied input voltage ramp $V_{\text {in }}$ and the measured memristor current $I_{\mathrm{mem}}$ (current limit $\mathrm{I}_{\mathrm{cc}}=600 \mathrm{uA}$ ) evolution with time during the 100 SET events. Red line shows the average current, whereas the 100 current curves are shown in gray color. (d), (e) show the resistance evolution w.r.t. the applied voltage and the flowing current, respectively, during the same 100 SET events.

For example, Fig. 3(b) demonstrates the resistance evolution w.r.t. energy for the same experiments, whereas in Fig. 3(d, e) we show the resistance evolution w.r.t. the applied voltage and the flowing current, respectively. In the latter, the curves result highly nonlinear, thus it is more difficult to figure out the quantitative effect of the applied voltage amplitude or the injected current on the resistive state of a device via a devised $\mathrm{d} R / \mathrm{d} V$ or $\mathrm{d} R / \mathrm{d} I$ parameter. On the other hand, in Fig. 3(d) we show how a linear approximation fit could be devised (based on the mean $d R / d E$ ) to describe the average energy required to reach to a specific resistive state, or vice versa: the final resistance achieved for a specific amount of injected energy. This is of course just an example to simplify the energy efficiency comparisons between different RS device types, even though more sophisticated mathematical expressions could be used to achieve a better fit to the $R$ - $t$ curve. Fig. 3(c) shows the voltage ramp $V_{\text {in }}$ applied to the top electrode of the memristor and the measured current $I_{\text {mem }}$ evolution with time during these 100 SET events. Note that the voltage drop on the memristor terminals is $V_{\mathrm{mem}}=V_{\mathrm{in}}-V_{\mathrm{x}}$ and is 


\section{REVISED Manuscript}

kept constant once the current limit is reached. $V_{\mathrm{x}}$ is the virtual ground voltage at the inverting input of the op-amp which, after saturation, it increases with $V_{\text {in }}$ accordingly to keep constant $V_{\text {mem }}$ [26]. Likewise, apart from the Knowm Inc. SDC commercial memristors, we also did experimental measurements on noncommercial bipolar RS devices with a completely different material stack, to check if the same trends are observed. More specifically, using an Agilent 4156C semiconductor parameter analyzer (SPA) and oscilloscope we performed 100 RESET-SET cycles on $5 \times 5 \mu \mathrm{m}^{2}$ $\mathrm{TiN}(200) / \mathrm{Ti}(10) / \mathrm{HfO}_{2}(10) / \mathrm{W}(200)(\mathrm{nm})$ bipolar RS devices (for details on device fabrication and experimental setup, see [29]). The devices were RESET always with the application of a negative ramp of specific maximum limit. Then, during every SET cycle we computed the energy consumed until the device overshot $\mathrm{R}_{\mathrm{INIT}} / 2$ (arbitrary SET criterion), i.e. half the initial resistance, and calculated the total resistance change per energy unit.

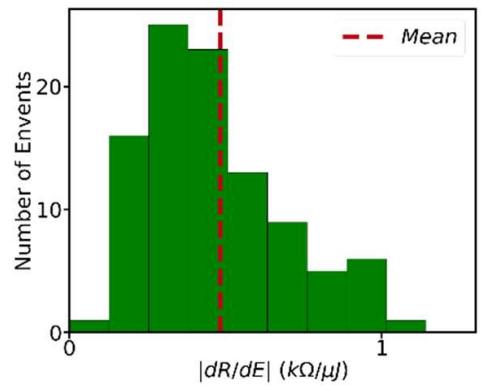

(a)

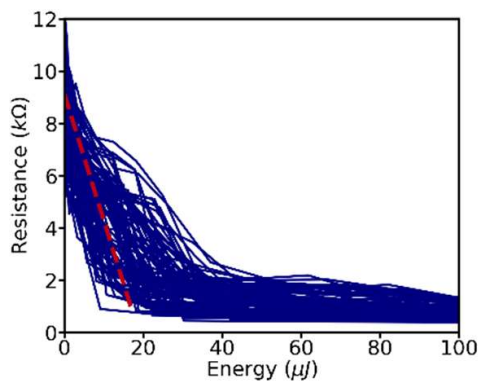

(b)

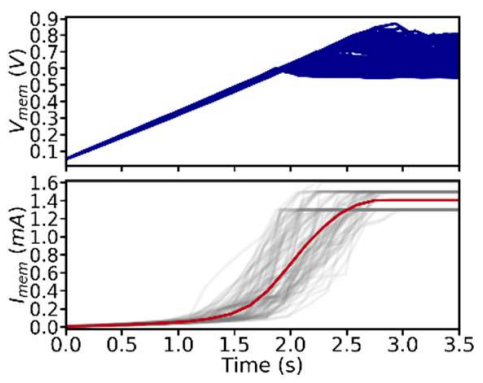

(c)

Fig. 4 Performance parameter analysis for analog operation of bipolar $\mathrm{HfO}_{2}$-based memristors under ramp voltage. (a) Histogram of 100 SET events depending on the resistance change per energy unit $(d R / d E)$ value. The vertical dashed line shows the mean value. (b) The corresponding resistance evolution w.r.t. injected energy during the same 100 SET events, applying a $0.3 \mathrm{~V} / \mathrm{s}$ VRS. The dashed line shows a linear approximation fit, based on the extracted $d R / d E$ parameter from (a). (c) The voltage drop on the memristor terminals $V_{\text {mem }}$ and the measured memristor current $I_{\text {mem }}$ evolution with time during the 100 SET events (current limit: 50 cycles with $\mathrm{I}_{\mathrm{cc}}=1.3 \mathrm{~mA}$ and 50 cycles with $\mathrm{I}_{\mathrm{cc}}=1.5 \mathrm{~mA}$ ). Red line shows the average current, whereas the 100 current curves are shown in gray color. $V_{\mathrm{mem}}$ is limited once the current $I_{\mathrm{mem}}$ reaches $\mathrm{I}_{\mathrm{cc}}$.

We present the results in Fig. 4 exactly in the same manner as in Fig. 3(a-c). We observe similar trends as the $d R / d E$ values are again concentrated around a mean value. From Fig. 3(b) and Fig. 4(b) it can be confirmed that the devices are operated in almost the same resistance window, and that the second device type demonstrates a more smooth switching behavior during a far more slowly rising applied ramp voltage, shown in Fig. 4(c). However, observing Fig. 3(a) and Fig. 4(a), from which the $d R / d E$ parameter is extracted, gives us some hints about the energy efficiency of the devices. An average $d R / d E$ of approximately $1.7 \mathrm{k} \Omega / \mathrm{nJ}$ against $0.5 \mathrm{k} \Omega / \mathrm{uJ}$ in our case indicates that switching the resistive state seems to cost less energy in the Knowm Inc. RS devices.

Therefore, the switching behavior of the devices under test can be comprehensively described when energy is the common denominator instead of time. We further observe this in Fig. 5 which presents the same data shown previously in Fig. 1(a) but now under a resistance change-rate perspective; i.e. the resistance evolution w.r.t. time in (a) and w.r.t. energy in (b), during the SET process, for different amplitudes of the applied square pulse. Each subfigure is composed of two plots; that on the left side shows the resistance evolution, whereas that on the far right side of each one shows the change-rate of the resistance $(d R / d t$ and $d R / d E$, respectively) w.r.t. the resistance during the SET process. By observing Fig. 5(a), we notice the following: (i) $d R / d t$ tends to increase with the increasing amplitude 


\section{REVISED Manuscript}

of the applied pulses, (ii) $d R / d t$ depends on the actual resistance of the device; the lower the resistance, the slower the switching rate, and (iii) higher pulse amplitudes drive the device deeper into a lower resistive state and the switching process seems to last longer. Nevertheless, there is a significant dispersion in the obtained data for a particular resistance value, which makes difficult the identification of any common trends in the overall behavior.

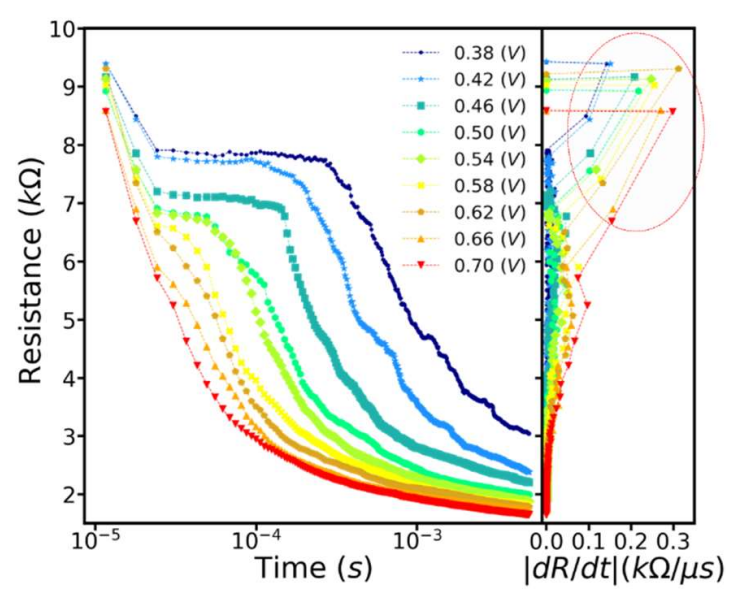

(a)

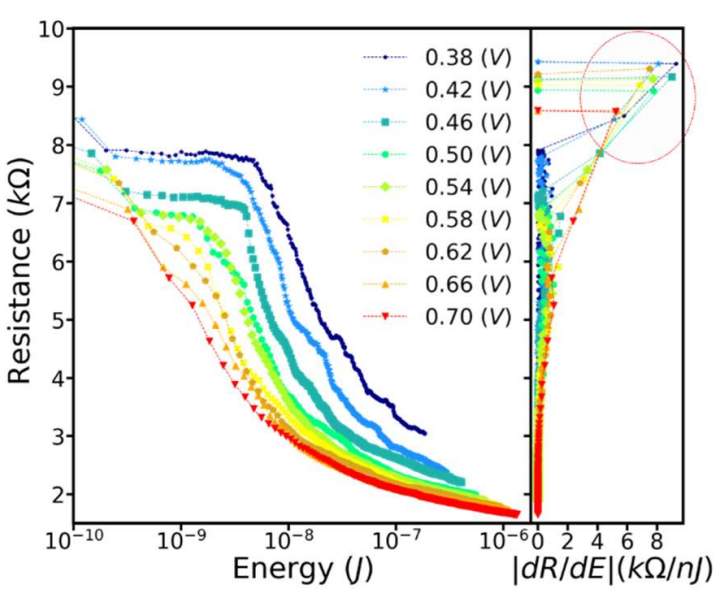

(b)

Fig. 5 Average resistance evolution analysis using data from Fig. 1(a). Resistance is shown in (a) w.r.t. time (pulse duration) and in (b) w.r.t. the injected energy. The right side of both sub-figures shows the average resistance change-rate w.r.t. time $(d R / d t)$ and w.r.t. the energy $(d R / d E)$, computed for every resistance value during the switching process. The circled areas are just a guide to the eye. Figures adapted from [24].

On the other hand, when the same analysis is done w.r.t. the computed energy, as shown in Fig. 5(b), the results are very much unified. Likewise in Fig. 5(a), here $d R / d E$ again depends on the actual resistance value $R$ and switching slows down as the device approaches to its low resistive state. However, for any given $R$ the average resistance change per energy unit $(d R / d E)$ is almost the same, thus being independent of the properties of the applied pulse. Such results indicate that the proposed $d R / d E$ performance parameter shows some evidence about the quantitative effect of the injected energy on the resistance, and it could be considered suitable to characterize analog RS device families.

Moreover, by observing the average resistance evolution with the injected energy as shown in Fig. 6, we realize that the cumulative effect of a specific amount of energy on the state of the device is on average the same, regardless of the way the energy is delivered. More specifically, Fig. 6 shows three curves, each one corresponding to a different pulsing scheme; i.e. a single 1-ms wide pulse, four consecutive 0.25 -ms wide pulses, or eight consecutive 0.125 -ms wide pulses, all having the same 0.5 $\mathrm{V}$ amplitude and the same 1-ms total duration. Each one of the three curves concerns the average of ten experiments, whereas the vertical lines indicate the entire range of resistance values measured for every value of energy. The device was always RESET above $6 \mathrm{k} \Omega$ before every SET process. We notice that the final state of the device tends to saturate in-between 1-2 $\mathrm{k} \Omega$ with a very similar average behavior during the entire process, which is independent of the applied pulsing scheme. Hence, we conclude that the energy justifiably qualifies as a magnitude capable of characterizing a specific memristive device family via the $d R / d E$ universal performance parameter. 


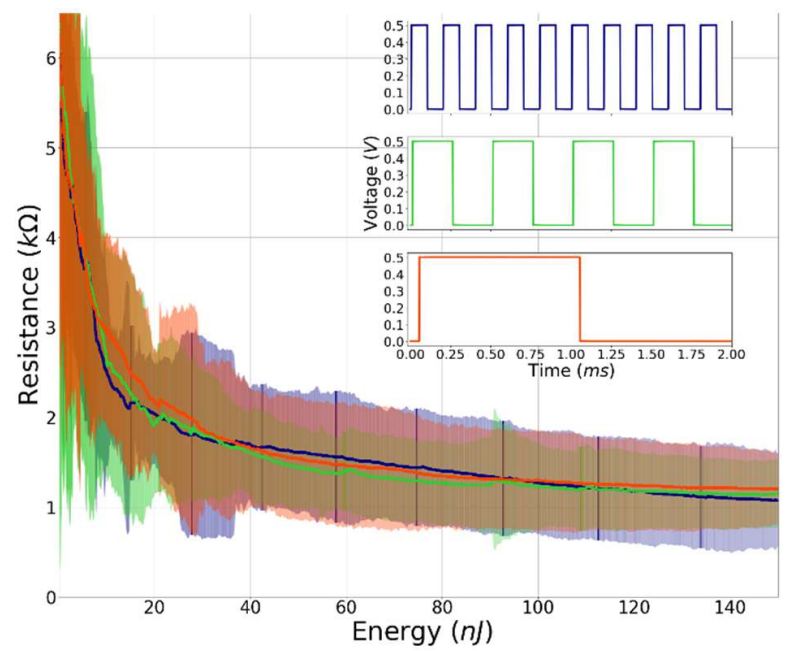

Fig. 6 Average resistance evolution w.r.t. the injected energy, when the latter is delivered through different pulsing schemes, shown in the inset. The device was RESET always above $6 \mathrm{k} \Omega$. Each curve shows the mean value of 10 experiments, whereas the vertical lines show the entire resistance range for every energy value. The curves are colored in correspondence with the colors in the inset.

\section{Energy-based Resistance Switching Model}

By observing the $R-E$ evolution in Fig. 5(b), we notice that for lower amplitudes (corresponding to slowly increasing ramps when the ramp rise time is fixed) there is an energy interval at the beginning of the SET process when the resistance curve remains almost horizontal, meaning that the device state is hardly changing at that moment. Therefore, during this interval there is an amount of energy delivered to the device practically without any impact on its resistance. We mentioned this previously in section 2.3 while observing the results in Fig. 2. We believe the same happens during the RESET process, where in fact this energy is much higher since the device is initially in low resistance. It is for this reason why we believe that the average energy measured until the RESET is complete, grows higher for lower VRS values, whereas for the SET process this effect is imperceptible. Therefore, we developed a simplified analytic model for the RESET process, assuming the following (see Fig. 7):

i. the excitation signal $\mathrm{V}(\mathrm{t})$ is a voltage ramp from 0 to $\mathrm{V}_{\mathrm{dd}}$ with rise time $\mathrm{T}$.

ii. the resistance of the device $\mathrm{R}(\mathrm{t})$ changes from a low resistive state $\left(\mathrm{R}_{\mathrm{ON}}\right)$ to a high resistive state ( $\left.\mathrm{R}_{\mathrm{OFF}}\right)$; we use a linear transition for simplicity; however, the $\mathrm{R}(\mathrm{t})$ expression can be optimized based on experimental data, so that the proposed approach can adapt better to other resistive switching devices with nonlinear transition $\mathrm{R}(\mathrm{t})$.

iii. the low resistive $\left(\mathrm{R}_{\mathrm{ON}}\right)$ and high resistive $\left(\mathrm{R}_{\mathrm{OFF}}\right)$ states demonstrate Ohmic behavior, without loss of generality, since any nonlinearities in these states (such as in valence change mechanism (VCM)-type devices [30]) will not affect the final outcome.

iv. the total energy $\mathrm{E}$ absorbed by the device has two components: a Joule heating component $\mathrm{E}_{\mathrm{Joule}}$ and a fixed component $\mathrm{E}_{\mathrm{RS}}=\alpha$, which is related to the resistance switching (RS).

So the mathematical expressions for $\mathrm{V}(\mathrm{t})$ and $\mathrm{R}(\mathrm{t})$ during the ramp are:

$V(t)=\left(\frac{V_{d d}}{T}\right) \cdot t=k \cdot t$ 
$R(t)=R_{O N}+\left(\frac{R_{O F F}-R_{O N}}{T}\right) \cdot t=m+n \cdot t$

where $k=\mathrm{V}_{\mathrm{dd}} / \mathrm{T}, m=\mathrm{R}_{\mathrm{ON}}$ and $n=\left(\mathrm{R}_{\mathrm{OFF}}-\mathrm{R}_{\mathrm{ON}}\right) / \mathrm{T}$. The expression for the total energy injected to the device during the application of the voltage ramp (duration $=\mathrm{T})$ is:

$E_{\text {total }}=E_{\text {Joule }}+E_{R S}=\int_{0}^{T} \frac{V^{2}(t)}{R(t)} d t+\alpha=\int_{0}^{T} \frac{k^{2} t^{2}}{m+n \cdot t} d t+\alpha$

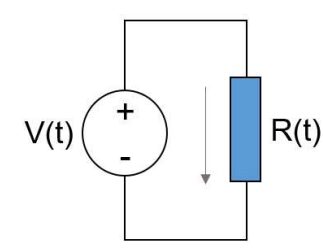

(a)

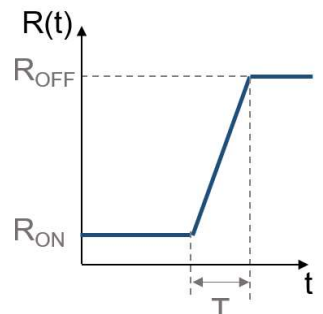

(c)

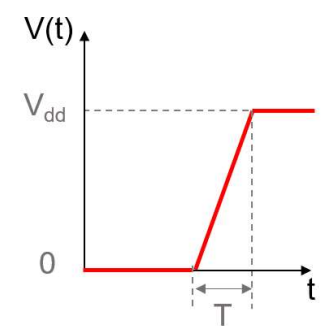

(b)

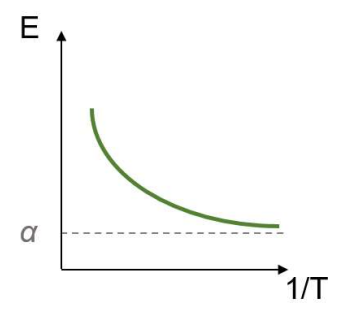

(d)

Fig. 7 Assumptions for the simplified analytic model of the energy consumed by the device in a RESET process. (a) A circuit schematic with a voltage source $V(t)$ connected to a memristor whose resistance is $R(t)$. (b) Graph showing the form of the applied excitation signal (voltage ramp from 0 to $V_{d d}$ with rise time $T$ ). (c) Graph showing a simplified evolution of the resistance $\mathrm{R}(\mathrm{t}$ ), assuming a linear RON to ROFF transition that occurs during the ramp rise time $\mathrm{T}$. (d) A cartoon plot for the expected form for the graph of the total energy E w.r.t. to 1/T according to (3) (based on Fig. 2).

$E_{\text {Joule }}$ corresponds to the energy dissipated in the time-varying resistance of the memristor during the voltage excitation in the time period $\mathrm{T}$. It is known that Joule heating plays an important role in the switching mechanism of some devices; for instance, RESET was identified as a thermally activated process in [31] for NiO-based devices. Temperature will rise owing to $E_{\text {Joule }}$ and this can affect the dynamics of the evolution of the creation/modification of the conductive filament. However, it is the $E_{R S}=\alpha$ energy component that is principally related to the internal structural modifications in the device implied by the resistance switching. The analytical solution of (3), whose plot w.r.t. 1/T (i.e. a magnitude proportional to VRS) shows the same trends with the real data for average energy consumption and asymptotically approaches $\mathrm{E}_{\mathrm{RS}}=\alpha$, as shown in Fig. 7(d). However, even though $\mathrm{T}$ is the rise time of every voltage ramp, the real upper limit of the integral in (3) is not $T$ in our case. In fact, it is lower than $\mathrm{T}$, since we calculate the energy delivered to the device only until it overshoots the 3-k $\Omega$ resistance threshold that we defined as a criterion for RESET. So, in order to fit (3) to our experimental data, we used parameter values $\mathrm{R}_{\mathrm{ON}}$ and $\mathrm{R}_{\mathrm{OFF}}$ that correspond to the commercial Knowm Inc. SDC memristor devices and for every VRS we extracted the correct "time to RESET" to be used 


\section{REVISED Manuscript}

in the integral. The difference between the rise time $\mathrm{T}$ of the applied voltage ramp and the real "time to RESET" used, is shown in Fig. 8(a), where we notice that for slowly rising ramps the device reached the $3-\mathrm{k} \Omega$ threshold much earlier than $\mathrm{T}$, i.e. before the ramp reached the maximum value.

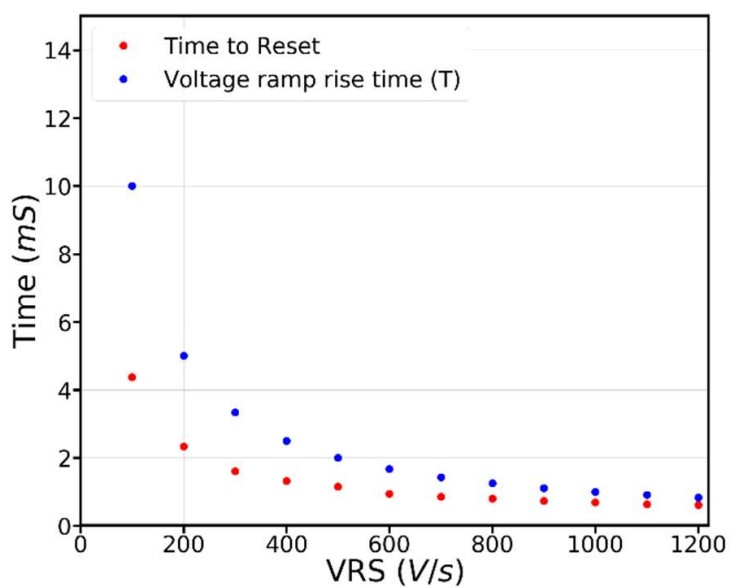

(a)

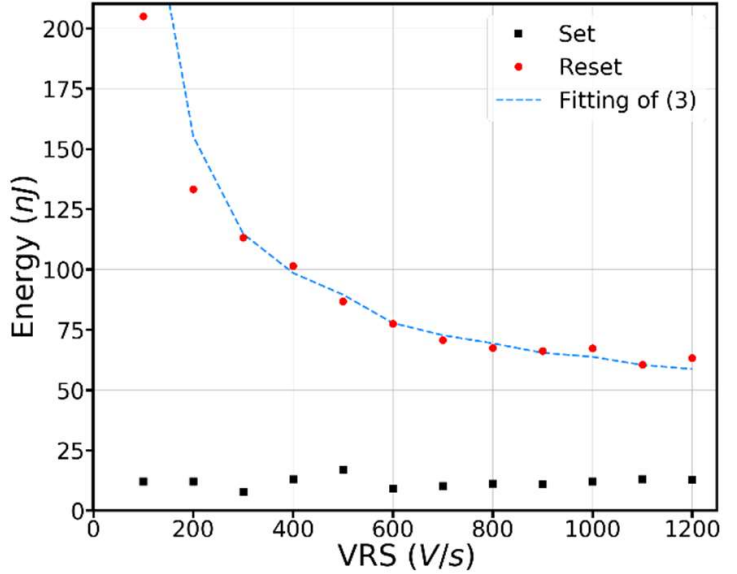

(b)

Fig. 8 (a) Values of T (rise time of voltage ramp) and average "time to RESET" (i.e. time until the device overshot $3 \mathrm{k} \Omega$ ) used as upper limit in the integral of (3), calculated for every VRS in our experimental data. (b) Fig. 2 plotted again with the theoretical values of ( 3 ) fitted to the experimental data for the RESET process.

Everything considered, in Fig. 8(b) we show again Fig. 2 but now including the curve-fit of (3) to the experimental data. We observe that the results agree with our initial conjectures. Parameter $\alpha$ works as a vertical shift of (3). We achieved the best fit using $\alpha=25 \mathrm{~nJ}$. However, the minimum value we notice in real data with VRS up to $1200 \mathrm{~V} / \mathrm{s}$ is approximately equal to 60nJ. This suggests that by further increasing the VRS (i.e. with a shorter rise time of the applied signal), the average energy consumed by the device should decrease further. As T approaches to zero, the rise time becomes null, and so does $E_{\text {Joule }}$, with the total energy consumed being dominated by $\mathrm{E}_{\mathrm{RS}}=\alpha$. Note also that the best fit for $\alpha$ is very close to the average energy consumed for the SET process. This observation suggests that the average $E_{R S}$ energy required to RESET the device ( $R_{O N} \rightarrow R_{O F F}$ ) is theoretically approximately the same with that required to SET it ( $\mathrm{R}_{\mathrm{OFF}} \rightarrow \mathrm{R}_{\mathrm{ON}}$ ). Further experimentation with much shorter RESET times is necessary to confirm such claim, which is beyond the scope of this work.

\section{Conclusions}

We explored experimentally from the energy point of view the SET-RESET behavior of both commercial SDC bipolar RS devices by Knowm Inc. and noncommercial $\mathrm{HfO}_{2}$-based samples. The energy was proven a much more comprehensive magnitude than the applied pulse amplitude and length. Moreover, energy is a magnitude that can be physically explained, given that filling the conductive channels of the SDC memristor with $\mathrm{Ag}+$ ions requires energy. Particularly, the "resistance change per energy unit" $(d R / d E)$ qualified not only for a better behavioral analysis but also as a universal performance parameter, suitable to characterize different RS device families in terms of energy efficiency, required for modern memory and computing applications. While exploring the quantitative result of the injected energy on the device state, we confirmed the observed trends in data taken on totally different bipolar RS devices and also devised a simplified mathematical 


\section{REVISED Manuscript}

model, to the best of our knowledge being the first of its kind, that approximates the energy delivered to the device during resistance switching with very good quantitative matching to experimental data. Certainly, an overall assessment of the energy consumption at circuit-level would also depend on the consumption by the driving circuitry.

\section{Acknowledgment}

The authors thank Knowm Inc. for providing the memristor samples used in our experiments. This work was supported by "Becas Iberoamerica Santander Universidades convocatoria 2018"; by the Chilean research grants CONICYT FONDECYT INICIACION No. 11180706 and CONICYT BASAL FB0008; by the Spanish MINECO and ERDF (TEC2016-75151-C3-R); and by the ICTS Network MICRONANOFABS.

\section{Declaration of Interest}

None.

\section{References}

[1] Y. V. Pershin and M. Di Ventra, "Practical approach to programmable analog circuits with memristors," IEEE Trans. Circuits Syst. I, Reg. Papers, vol. 57, no. 8, pp. 1857-1864, Aug. 2010

[2] MemComputing Inc., "Powerful Co-Processors with Speed like no other," [Online]. Available: http://memcpu.com [Accessed November 2018].

[3] M. A. Nugent, T. W. Molter, "Thermodynamic-RAM technology stack," Int. Journal of Parallel, Emergent and Distributed Systems, vol. 33, no. 4, pp. 430-444, 2018

[4] V. Ntinas, I. Vourkas, and G. Ch. Sirakoulis, "LC Filters with Enhanced Memristive Damping," 2015 IEEE Int. Symp. Circ. Syst. (ISCAS 2015), Lisbon, Portugal, May 24-27, pp. $2664-2667$

[5] Y. V. Pershin, S. N. Shevchenko, and F. Nori, "Memristive Sisyphus circuit for clock signal generation," Sci. Rep., vol. 6, no. 26155, 2016

[6] L. O. Chua, "Memristor - The Missing Circuit Element," IEEE Trans. Circuit Theory, vol. 18, no. 5, pp. 507-519, 1971

[7] D. B. Strukov, G. S. Snider, D. R. Stewart, and R. S. Williams, "The missing memristor found," Nature, vol. 453, pp. 80 - 83, 2008

[8] R. Williams, "How we found the missing memristor," IEEE Spectrum, vol. 45, no. 12, pp. 28-35, Dec. 2008

[9] P. Rabbani, R. Dehghani, and N. Shahpari, "A multilevel memristor-CMOS memory cell as a ReRAM," Microelectronics J., vol. 46, no. 12-A, pp. 1283-1290, 2015

[10] G. Papandroulidakis, I. Vourkas, A. Abusleme, G. Ch. Sirakoulis, and A. Rubio, "Crossbar-Based Memristive Logic-In-Memory Architecture”, IEEE Trans. Nanotechnol., vol. 16, no. 3, pp. 491 - 501, 2017

[11] M. Prezioso, et al., "Spike-timing-dependent plasticity learning of coincidence detection with passively integrated memristive circuits," Nature Comm., vol. 9(5311), 14 December 2018

[12] H. Li, T. F. Wu, S. Mitra, and H.-S. P. Wong, "Resistive RAM-centric computing: Design and modeling methodology," IEEE Trans. Circuits Syst. I, Reg. Papers, vol. 64, no. 9, pp. 2263-2273, Sep. 2017

[13] C. Yakopcic, T. M. Taha, G. Subramanyam, and R. E. Pino, "Memristor SPICE Modeling," in Advances in Neuromorphic Memristor Science and Applications, 1st Ed. The Netherlands: Springer, 2012, pp. 211-244

[14] Z. Jiang, et al., "A Compact Model for Metal-Oxide Resistive Random Access Memory with Experiment Verification," IEEE Trans. Electron Devices, vol. 63, no. 5, pp. 1884-1892, 2016

[15] S. Menzel, U. Böttger, M. Wimmer, and M. Salinga, "Physics of the Switching Kinetics in Resistive Memories," Adv. Funct. Mater., vol. 25, pp. 6306-6325, 2015 


\section{REVISED Manuscript}

[16] Knowm Inc., “Neuromemristive Artificial Intelligence," [Online]. Available: https://knowm.org

[17] Y. V. Pershin and M. Di Ventra, "Memory effects in complex materials and nanoscale systems," $A d v$. Phys., vol. 60, no. 2, pp. 145-227, 2011

[18] H.-S. P. Wong, et al., "Metal-Oxide RRAM,” IEEE Proc., vol. 100, no. 6, pp. 1951-1970, 2012

[19] M. Villena, J. Roldán, F. Jiménez-Molinos, E. Miranda, J. Suñé, and M. Lanza, "SIM²RRAM: a physical model for RRAM devices simulation,” J. Comput. Electron., vol. 16, no. 4, pp. 1095-1120, 2017

[20] G. Sassine, et al., "Interfacial versus filamentary resistive switching in $\mathrm{TiO} 2$ and $\mathrm{HfO} 2$ devices," $J$. Vac. Sci. Technol. vol. B34 (012202), 2016

[21] S. H. Jo, et al., "Programmable Resistance Switching in Nanoscale Two-Terminal Devices," Nano Lett., vol. 9, no. 1, pp. 496-500, 2009

[22] M. Maestro, et al., "Analysis of Set and Reset mechanisms in Ni/HfO2-based RRAM with fast ramped voltages," Microelectronic Eng., vol. 147, pp. 176-179, 2015

[23] J. Gomez, et al., "Universal Performance Parameters for Resistive Switching Devices," Late Break News session in 2017 IEEE Int. Symp. Circ. Syst. (ISCAS), Baltimore, MD, USA, May 28-31

[24] J. Gomez, I. Vourkas, A. Abusleme, and A. Rubio, "Resistive Switching Behavior seen from the Energy Point of View," 2018 IEEE Int. Symp. on On-Line Testing and Robust System Design (IOLTS), Platja d'Aro, Costa Brava, Spain, July 2-4

[25] Analog Discovery 2, "A high performance, all-in-one portable oscilloscope \& instrumentation system," [Online]. Available: https://analogdiscovery.com

[26] J. Gomez, I. Vourkas, A. Abusleme, and G. Ch. Sirakoulis, "Experimental Measurements on Resistive Switching Devices: Gaining Hands-on Experience," 2018 Int. Conf. on Modern Circuits and Syst. Technol. (MOCAST), Thessaloniki, Greece, May 07-09

[27] K. Campbell, "Self-directed channel memristor for high temperature operation," Microelectron. J., vol. 1, no. 1, pp. 10-14, 2017

[28] F. Alibart, L. Gao, B. D. Hoskins, and D. B. Strukov, "High precision tuning of state for memristive devices by adaptable variation-tolerant algorithm," Nanotechnology, vol. 23 (075201), 2012

[29] M. Pedro, et al., "Tuning the conductivity of resistive switching devices for electronic synapses", Microelectronic Eng., vol.178, pp. 89-92, 2017

[30] F. Cüppers, S. Menzel, C. Bengel, A. Hardtdegen, M. von Witzleben, U. Böttger, R. Waser, and S. Hoffmann-Eifert, "Exploiting the switching dynamics of HfO2-based ReRAM devices for reliable analog memristive behavior", APL Mater., vol. 7 (091105), 2019

[31] U. Russo, D. Ielmini, C. Cagli, and A. Lacaita "Filament Conduction and Reset Mechanism in NiOBased Resistive-Switching Memory (RRAM) Devices”, IEEE Transactions on Electron Devices, vol. 56, no. 2, pp. 186-192, 2009 